[8] A. Kralj, R. J. Jaeger, and M. Munih, "Analysis of standing up and sitting down in humans: Definitions and normative data presentation," J. Biomechan., vol. 23, pp. 1123-1138.

[9] R. S. W. Nash, M. S. Davy, R. Orpwood, and I. D. Swain, "Development of a wheelchair-mounted folding frame," J. Biomed. Eng., vol. 12, pp. 189-192, 1990.

[10] T. A. Perkins, N. de N. Donaldson, A. C. Worley, V. Harper, D. E. Wood, and D. N. Rushton, "Initial results with a lumbar/sacral anterior root stimulator implant," in Neuroprosthetis: From Basic Reserch to Clinical Application, A. Pedotti, M. Ferrarin, J. Quintern, and R. Riener, Eds.New York: Springer-Verlag, pp. 623-634.

[11] G. F. Phillips, J. A. Adler, and S. G. J. Taylor, "A portable programmable stimulator for surface FES," in Proc. Ljubljana FES Conf., R. Jaeger and T. Bajd, Eds., 1993, pp. 166-168.

[12] R. Riener and T. Fuhr, "Patient-driven control of FES-supported standing-up: A simulation study," this issue, pp. 113-124.

[13] A. B. Schultz, N. B. Alexander, and J. A. Ashton-Miller, "Biomechanical analyzes of rising from a chair," J. Biomechan., vol. 25, pp. 1383-1391, 1992

[14] C.-H. Yu and N. Donaldson, "Disability and controllability after paralysis: A kinematic analysis," in Proc. Int. FES Soc. Conf., Vancouver, B.C., Canada, Aug. 1997, pp. 195-196.

[15] _ "Measurement of posture by triangulation using potentiometers," Med. Eng. Phys., to be published.

\section{Switching Curve Controller for FES-Assisted Standing Up and Sitting Down}

Michael J. Dolan, Brian J. Andrews, and Peter H. Veltink

Abstract-A low-level, closed-loop controller for FES-assisted standing up and sitting down is described. If, for able-bodied individuals, when standing up and sitting down, the knee angular velocity is plotted against knee angle, consistent phase-plane trajectories are produced. The bangbang controller uses a model of this trajectory as a switching curve. The design rationale for the controller was the desire to avoid injuries that might occur if knee-locking on standing up and seat-contact on sitting down are not adequately controlled. This switching curve controller (SCC) was incorporated within a hierarchical, finite state control scheme, with electrical stimulation applied bilaterally to the knee extensors. The SCC was tested in a pilot study on a female volunteer with paraplegia (T5/6 ASIA A) and evaluated against an unramped, open-loop controller (OLC). The vertical hand forces and knee angles were measured. The subject was able to achieve standing up and sitting down safely using both controllers. For standing up, the SCC was not found to offer any quantifiable advantages over the OLC and was found to increase the hand force by $8.4 \%$. In contrast, for sitting down the SCC was found to reduce the knee angular velocities as the subject approached the seat by $27 \%$, demonstrating a safer, softer landing.

\section{INTRODUCTION}

Standing up and sitting down are prerequisites for independent standing and upright mobility. These frequently executed maneu-

Manuscript received November 25, 1997; revised February 1, 1998 and March 17, 1998. M. J. Dolan was supported by the United Kingdom Science and Engineering Research Council and the European Union ERASMUS programme.

M. J. Dolan is with the Department of Orthopaedic and Trauma Surgery, University of Dundee, Dundee DD1 4HN U.K.

B. J. Andrews is with the Department of Biomedical Engineering, University of Alberta, Edmonton, Alta. T6G 2G3 Canada.

P. H. Veltink is with the Department of Electrical Engineering, University of Twente, 7500 AE Enschede, The Netherlands.

Publisher Item Identifier S 1063-6528(98)04307-9.

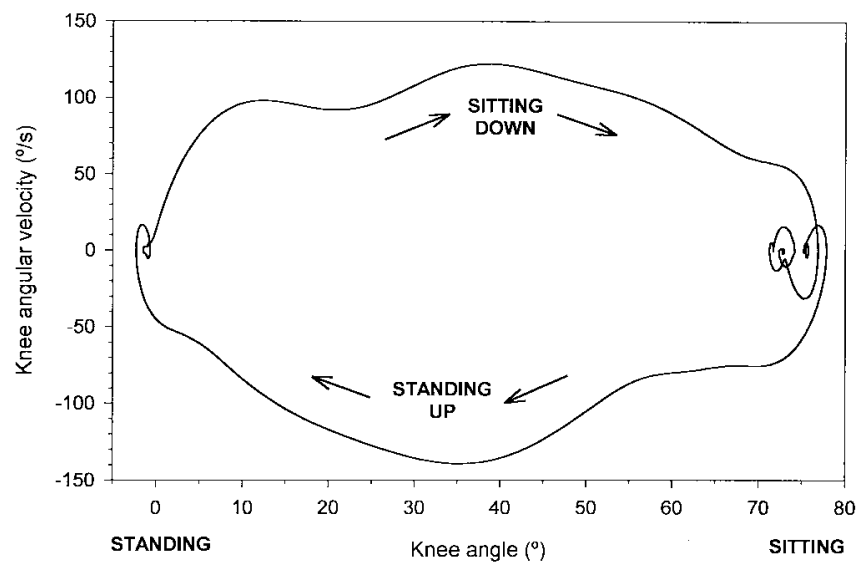

Fig. 1. Typical graph of knee angular velocity against angle trajectory for able bodied individuals during standing up and sitting down [2]. Full extension was defined as $0^{\circ}$ and flexion as positive, hence the angular velocity was negative during standing up as the knee angle reduced.

vers are also, in some respects, two of the most biomechanically demanding activities routinely undertaken [1]. The standard technique employed by able bodied individuals to stand up from a sitting position is essentially a dynamic, sagittal plane maneuver, with the hips and trunk initially flexing to move the upper body over the base of support and to generate forward momentum, followed by extension of the lower limb joints to elevate the body to a standing position. Sitting down is also essentially a sagittal plane maneuver and can, to some extent, be regarded as the reverse of standing up. The hip and knee joints flex to lower the body in a controlled manner until the buttocks contact the seat. The hips and trunk are then extended to achieve a sitting position [2]. Similar techniques have been employed to stand persons with paraplegia with the assistance of functional electrical stimulation (FES) applied to the lower limbs. The maneuvers, however, tend to be less vigorous with the upper limbs used to supplement the lower limbs. Typically, electrical stimulation (ES) is applied bilaterally to the knee extensors to stand up and to control the flexion of the knees to sit down [3]-[6].

A low-level, bang-bang, closed-loop controller is presented, which is based partially on that originally described in [7]. For able-bodied subjects, the trajectories of knee angular velocity against knee angle are consistent over the range of durations found (Fig. 1). Using representative trajectories as switching curves, for stimulation applied to the knee extensors, should allow the knee velocity to be controlled over the full range of knee motion. This does not constrain the maneuvers to a fixed time duration as, in practice, the movement will not conform exactly to the specified curve. The primary objective of this switching curve controller (SCC) was to control the terminations of the maneuvers, i.e., the locking of the knee joints on standing and the contact with the seat on sitting, so as to protect the delicate ligaments of the knee joints and the insensate tissues of the buttocks from injury [8]. The secondary objective was to reduce the upper limb loads during the maneuvers so as not to exacerbate shoulder pain due to overuse [9]. Reduction in the upper limb loads would also allow FES standing to be extended to more frail, elderly and possibly obese persons with paraplegia, and to those with higher lesions, if used with FES trunk stabilization. The SCC was evaluated in the pilot study reported here. 


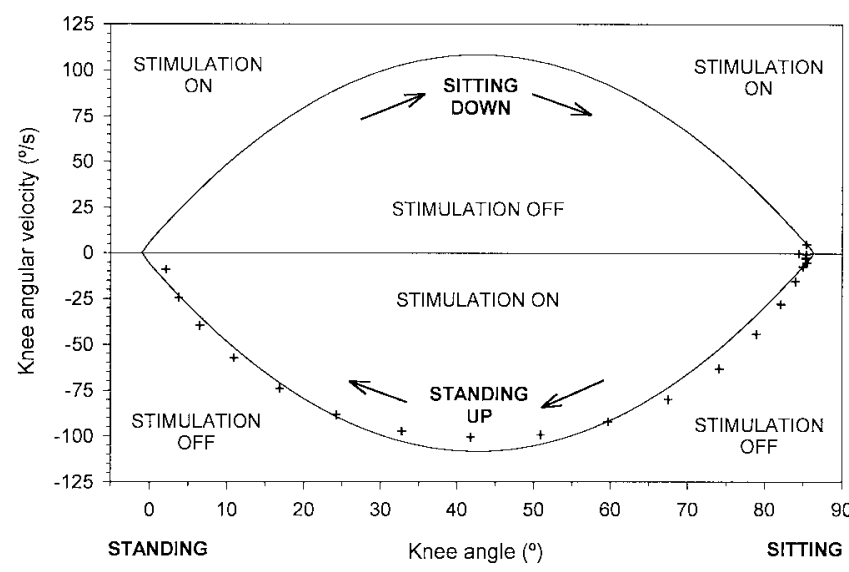

Fig. 2. Plot of knee angular velocity against angle data of [10] for standing up. The angular velocity data were determined by applying a numerical differentiator (three-point span) to the reported knee angle data. This is overlaid with the second-order polynomial model for standing up which was the basis for the switching curve for both standing up and sitting down. The stimulation status of the SCC for positions relative to the switching curve are indicated.

\section{METHODS}

\section{A. The Switching Curve Model}

The averaged knee angle data reported in a study of standing up in young, able-bodied individuals (17 male, 38 female) [10] was used to determine a suitable model of the angular velocity against angle trajectory (Fig. 2). The maximum angular velocity was found to be $-100.7^{\circ} / \mathrm{s}$ at $50.2 \%$ of the knee angle range and was comparable to that reported elsewhere [2], [11]. The trajectory was fitted with a second-order polynomial as a model for the switching curve ( $r$ value 0.986), i.e.

$$
\omega=a_{2} \theta^{2}+a_{1} \theta+a_{0}
$$

where $\omega$ is the knee angular velocity, $\theta$ is the knee angle and $a_{n}$ are the polynomial coefficients.

The curve was defined using three points. The angular velocities at the start and termination of standing up (at angles $\theta_{S}$ and $\theta_{T}$, respectively) must be zero, i.e.

$$
0=a_{2} \theta_{S}^{2}+a_{1} \theta_{S}+a_{0}, 0=a_{2} \theta_{T}^{2}+a_{1} \theta_{T}+a_{0} .
$$

The maximum angular velocity, $\omega_{\max }$, occurs at the midangle between $\theta_{S}$ and $\theta_{T}$ [2], thus

$$
\omega_{\max }=a_{2}\left[\frac{\theta_{S}+\theta_{T}}{2}\right]^{2}+a_{1}\left[\frac{\theta_{S}+\theta_{T}}{2}\right]+a_{0} .
$$

Equations for the polynomial coefficients were obtained by solving the simultaneous (2) and (3) giving

$$
\begin{aligned}
& a_{2}=-\frac{4 \omega_{\max }}{\left(\theta_{S}-\theta_{T}\right)^{2}} \\
& a_{1}=\frac{4 \omega_{\max }\left(\theta_{S}+\theta_{T}\right)}{\left(\theta_{S}-\theta_{T}\right)^{2}} \\
& a_{0}=\frac{4 \omega_{\max } \theta_{S} \theta_{T}}{\left(\theta_{S}-\theta_{T}\right)^{2}} .
\end{aligned}
$$

\begin{tabular}{|c|c|c|}
\hline State & Rule set & Rules \\
\hline \multirow{2}{*}{$\begin{array}{c}\text { s1 } \\
\text { (sitting) }\end{array}$} & Entry & Stimulation off. \\
\hline & Transit & IF $m$ THEN transit to $s 2$. \\
\hline \multirow{3}{*}{$\begin{array}{l}\text { s2 } \\
\text { (stand- } \\
\text { ing up) }\end{array}$} & Entry & $\begin{array}{l}\text { Calculate poly. coefficients. } \\
\text { Stimulation on. }\end{array}$ \\
\hline & Continual & $\begin{array}{l}\text { IF } \omega \geq \omega_{\mathrm{SC}}(\theta) \text { THEN stim. on } \\
\text { ELSE stimulation off. }\end{array}$ \\
\hline & Transit & $\begin{array}{l}\text { IF } \theta<\theta_{1} \text { THEN transit to } s 3 \\
\text { IF } m \text { THEN transit to } s 3 \text {. } \\
\text { IF } e \text { THEN transit to } s 1 \text {. }\end{array}$ \\
\hline \multirow{2}{*}{$\begin{array}{l}\text { s3 } \\
\text { (stand- } \\
\text { ing) }\end{array}$} & Entry & Stimulation on. \\
\hline & Transit & $\begin{array}{l}\text { IF } m \text { THEN transit to } s 4 \text {. } \\
\text { IF e THEN transit to } s 1 \text {. }\end{array}$ \\
\hline \multirow{3}{*}{$\begin{array}{l}\text { s4 } \\
\text { (sitting } \\
\text { down) }\end{array}$} & Entry & Stimulation off. \\
\hline & Continual & $\begin{array}{c}\text { IF } 0 \geq-\omega_{S C}(\theta) \text { THEN stim. on } \\
\text { ELSE stimulation off. }\end{array}$ \\
\hline & Transit & $\begin{array}{l}\text { IF } 0 \geq 0, \text { THEN transit to } s 1 \text {. } \\
\text { IF } m \text { or } e \text { THEN transit to } s 1 \text {. }\end{array}$ \\
\hline
\end{tabular}

Data were not available for sitting down, however, the trajectories for both maneuvers are similar (Fig. 1). Therefore the same secondorder polynomial model, except reflected in the zero angular velocity axis, was used for sitting down.

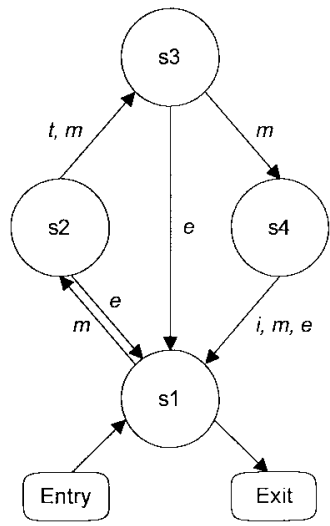

Fig. 3. State transition diagram and description table for the SCC. Transitions between states occurred when the manual override $(m)$ or the emergency (e) keys were depressed by the control computer operator, or the standing $(t)$ or sitting $(i)$ positions were detected.

\section{B. Synthesis of the Controllers}

The SCC was implemented within a hierarchical, finite state control scheme [5] consisting of four states (Fig. 3). The polynomial coefficients were calculated on entry to the standing up state (s2). For standing up, the start angle $\theta_{S}$ was that measured on entry to s2. The termination angle $\theta_{T}$ was assumed to be the angle at full extension. This was determined beforehand by manually holding the subject's knees at full extension and sampling the outputs from the electrogoniometers. For sitting down, the start and termination angles were assumed to be the reverse of those for standing up (subject returns to same sitting position [2]). The same maximum angular velocity $\omega_{\max }$ was chosen to optimize the control of ascent and descent. The transitions between states were controlled by the transit rule sets. The transition $(t)$ between $\mathrm{s} 2$ and the standing state (s3) was determined by the standing transition angle $\theta_{t}$ defined as $2^{\circ}$ of flexion. The transition (i) between the sitting down state (s4) and the sitting state $(\mathrm{s} 1)$ was determined by the sitting transition angle $\theta_{i}$ defined as equal to $\theta_{S}$. A manual override $(m)$ allowed both transitions to be implemented by the control computer operator. In $\mathrm{s} 2, \mathrm{~s} 3$, and $\mathrm{s} 4$ the operator could, if required, force a transition (e) to $\mathrm{s} 1$ and thereby terminate stimulation.

During states $\mathrm{s} 2$ and $\mathrm{s} 4$, the SCC routine was evoked by a computer interrupt operating at $100 \mathrm{~Hz}$. The collected knee angle data were digitally filtered using a moving average filter (five-point span). A decision was made every five interrupts whether or not stimulation pulses should be delivered to the two legs (limiting the stimulation frequency to a maximum of $20 \mathrm{~Hz}$ ). The angular velocity was calculated as the rate of change of the knee angle since the previous decision interrupt. Stimulation delivery was dependent on the knee angle position relative to the switching curve (Fig. 2). For example, if, during standing up, the knee was moving too slowly relative to the switching curve then a pulse was delivered. This decision making process was implemented using IF-THEN-ELSE rules (Fig. 3). For simplicity only the right knee angle was used as input to the controller. The switching curve threshold $\omega_{\mathrm{SC}}(\theta)$ was calculated (1) using the filtered knee angle and the polynomial coefficients.

The open-loop controller (OLC) was an implementation of the standard Ljubljana technique now widely used for FES-assisted standing up and sitting down with one channel of stimulation per leg [3]-[6]. The stimulation applied to the quadriceps was either completely on or completely off. The stimulation was controlled by the computer operator who followed verbal requests from the subject. For both controllers the stimulus intensity was preset. 
TABLE I

Averaged Results and Statistically Comparison for Standing Up with Stimulation. DF Is the Number of Degrees of Freedom. The Values Are the Average of Both Left and Right Sides and Given as Per Side. Able Bodied Data Taken from a Study of Ten Young Subjects Performing Standing Up and Sitting Down with Their Hands on the Handles of a Standing Frame [2]

\begin{tabular}{lccccr}
\hline & OLC & \multicolumn{1}{c}{ SCC } & DF & $p$ & Able bodied \\
\hline Duration (s) & $2.54 \pm 0.66$ & $3.78 \pm 0.75$ & 16 & $0.001<p \leq 0.01$ & $2.35 \pm 0.33$ \\
Max. ext. ang. vel. $(\% / s)$ & $-116.5 \pm 29.0$ & $-123.8 \pm 20.1$ & 34 & $p>0.05$ & $-111.3 \pm 34.5$ \\
Ang. vel. at $5^{\circ}(\% / s)$ & $-40.0 \pm 17.6$ & $-53.7 \pm 27.0$ & 34 & $p>0.05$ & $-40.4 \pm 16.6$ \\
Ang. vel. at $2^{\circ}(\% / s)$ & $-17.5 \pm 11.8$ & $-24.6 \pm 18.7$ & 34 & $p>0.05$ & $-24.9 \pm 11.2$ \\
Ang. vel. at $1^{\circ}(\% / s)$ & $-16.8 \pm 8.7$ & $-14.4 \pm 9.4$ & 34 & $p>0.05$ & $-16.4 \pm 8.0$ \\
Ang. acc. at full ext. $\left(\% / s^{2}\right)$ & $324 \pm 254$ & $292 \pm 304$ & 20 & $p>0.05$ & $130 \pm 110$ \\
Support force $(\mathrm{N})$ & $166 \pm 13$ & $180 \pm 21$ & 34 & $0.02<p \leq 0.05$ & \\
Stimulation duration $(\%)$ & $-2.3 \pm 2.0$ & $-1.7 \pm 1.1$ & 16 & $p>0.05$ & \\
\hline
\end{tabular}

\section{Equipment}

Flexible electrogoniometers (type M180, Penny and Giles Biometrics Ltd., Blackwood, U.K.) were mounted laterally over both knee joints. These were calibrated on a circular template and the zero offsets were determined when mounted with the knees held at full extension. The lateral, horizontal support bars of an adjustable, rigid, standing frame were instrumented with strain gauges (type CEA-06062UW-120, Micro-Measurements, Raleigh, NC) to determine the vertical force applied by the hands. The outputs were calibrated by suspending known masses. Calibration coefficients were determined using linear least-square-error regressions.

The stimulation system consisted of an optically isolated, batterypowered, eight-channel stimulator (current regulated, monophasic waveform) and an IBM compatible PC, self-adhesive, rectangular, surface electrodes were used (Pals Flex, Axelgaard Manufacturing Co. Ltd, Fallbrook, CA). The cathode was positioned over the motor points of the rectus femoris and vastus lateralis. The anode was placed proximal to the base of the patella, approximately over the motor point of the vastus medialis. The purpose-written computer program was used to simultaneously control the stimulator and collect data from a 12 bit A/D card at a sampling rate of $100 \mathrm{~Hz}$.

\section{Subject and Trials Performed}

One female volunteer (age 29, 9 years postinjury, mass $56 \mathrm{~kg}$ ) with a ASIA A complete T5/6 lesion of the spinal cord was recruited. She used ES for muscle conditioning three-four times per week for periods up to one hour. She had undergone a selection and training programme [12] and had experience of standing with an orthosis and with FES. She was able to achieve an isometric knee extension moment of $24 \mathrm{~N} \cdot \mathrm{m}$ with quadriceps stimulation (frequency $20 \mathrm{~Hz}$, current amplitude $100 \mathrm{~mA}$, pulse width $500 \mu \mathrm{s}$ ) at $60^{\circ}$ of flexion. The experimental protocol and informed consent form were approved by the Roessingh Rehabilitation Centre's Medical Ethics Committee.

The subject stood within the standing frame using her upper limbs to assist her ascent and control her descent. The support bars were adjusted for the subject (height $89 \mathrm{~cm}$, gap $54 \mathrm{~cm}$ ) and her preferred hand positions were marked and used throughout. The subject performed 22 stand-sit maneuvers. Initially a set of three trials were performed without stimulation. One test trial was performed with stimulation to assist with optimizing the current amplitude for the OLC. Six sets of three trials were performed with stimulation (first OLC, four SCC, and final OLC). The principal stimulation frequency was $20 \mathrm{~Hz}$, but this was effectively varied by the SCC. The current amplitude was $70 \mathrm{~mA}$ and the pulse width was $500 \mu \mathrm{s}$. The maximum angular velocity, which defined the switching curve peak at the midangle (3), was varied between -100 and $-140^{\circ} / \mathrm{s}$ in an attempt to optimize performance.

\section{E. Data Analysis for Evaluation Purposes}

For evaluation purposes, the original data were low-pass filtered using a fourth-order Butterworth digital filter with a cutoff frequency of $5 \mathrm{~Hz}$ to maintain fidelity over the signal bandwidths. Numerical differentiators (seven-point span) were applied to the electrogoniometer data to determine the knee angular velocities and accelerations [13].

The durations of the maneuvers were determined using the start and termination events which were defined as the time at which the magnitude of the angular velocities exceeded or settled below $2 \%$, respectively [2]. For standing up, the maximum extension angular velocity and the angular velocities as the knees approached the maximum extension angle achieved in the maneuver (at within 5,2 , and $1^{\circ}$ of maximum extension) were determined. The angular acceleration at the maximum extension angle was also determined (except when the maximum extension angle was at the termination of the maneuver). Likewise, for sitting down the maximum flexion angular velocity and angular velocities as the knee approached the maximum flexion angle were determined, as was the angular acceleration at the maximum flexion angle. The mean support forces were determined between the start and termination events. The stimulation duration prior to the start of standing up, and the stimulation duration after the start of sitting down, were determined as percentages of the maneuver durations. The values of these parameters obtained for the two controllers were compared statistically using the paired and two-sample $t$-tests. A probability level of $p \leq 0.05$ was accepted as indicative of a statistically significant difference.

\section{RESULTS}

The subject was able to stand up and sit down without stimulation using her upper body strength alone. The mean support forces for the three trials without stimulation were $266 \pm 13$ (std dev.) and $179 \pm 24 \mathrm{~N}$ per side, for standing up and sitting down, respectively. As expected, she was neither able to fully extend her knees nor support her weight through her lower limbs once standing (support force $279 \pm 13 \mathrm{~N}$ ). With stimulation (both controllers) the support forces were significantly reduced for standing up, by $91 \mathrm{~N}(p \leq$ $0.001)$, and for standing, by $186 \mathrm{~N}(p \leq 0.001)$, indicating that the subject was better able to bear weight on her lower limbs.

The subject was able to reliably stand up and sit down using both controllers. With stimulation (both controllers), the support forces were significantly less $(p \leq 0.001)$ during standing than during standing up and sitting down, by $83 \mathrm{~N}$ and $61 \mathrm{~N}$, respectively. For both controllers the general trend was for the angular velocity to decrease as the knees approach the maneuver terminations (Tables I and II). With the SCC some oscillation was found to occur at the start and termination of standing up and to a lesser extent at the start of sitting down (Fig. 4). This was thought to be due to the low angular velocity threshold at the extremes of the maneuvers (Fig. 2) 
TABLE II

Averaged Results and Statistically Comparison for Sitting Down with Stimulation. Able Bodied Data from [2]

\begin{tabular}{lccccr}
\hline & OLC & \multicolumn{1}{c}{ SCC } & DF & $p$ & Able bodied \\
\hline Duration (s) & $2.72 \pm 0.73$ & $2.44 \pm 0.46$ & 16 & $p>0.05$ & $2.79 \pm 0.51$ \\
Max. flex. ang. vel. $(\% / s)$ & $201.8 \pm 53.0$ & $170.9 \pm 47.6$ & 34 & $p>0.05$ & $101.7 \pm 29.5$ \\
Ang. vel. at $5^{\circ}(\% / s)$ & $125.6 \pm 29.5$ & $94.1 \pm 44.9$ & 34 & $0.02<p \leq 0.05$ & $47.7 \pm 17.6$ \\
Ang. vel. at $2^{\circ}(\% / s)$ & $80.6 \pm 25.5$ & $58.5 \pm 29.7$ & 34 & $0.02<p \leq 0.05$ & $32.2 \pm 18.1$ \\
Ang. vel. at $1^{\circ}(\% / s)$ & $55.3 \pm 17.2$ & $39.7 \pm 20.7$ & 34 & $0.02<p \leq 0.05$ & $21.3 \pm 14.0$ \\
Ang. acc. at full flex. $\left(\% / \mathrm{s}^{2}\right)$ & $-1773 \pm 1086$ & $-1200 \pm 1005$ & 33 & $p>0.05$ & $-503 \pm 415$ \\
Support force $(\mathrm{N})$ & $143 \pm 64$ & $158 \pm 38$ & 34 & $p>0.05$ & \\
Stimulation duration $(\%)$ & $0.4 \pm 5.8$ & $50.1 \pm 15.8$ & 16 & $p \leq 0.001$ & \\
\hline
\end{tabular}

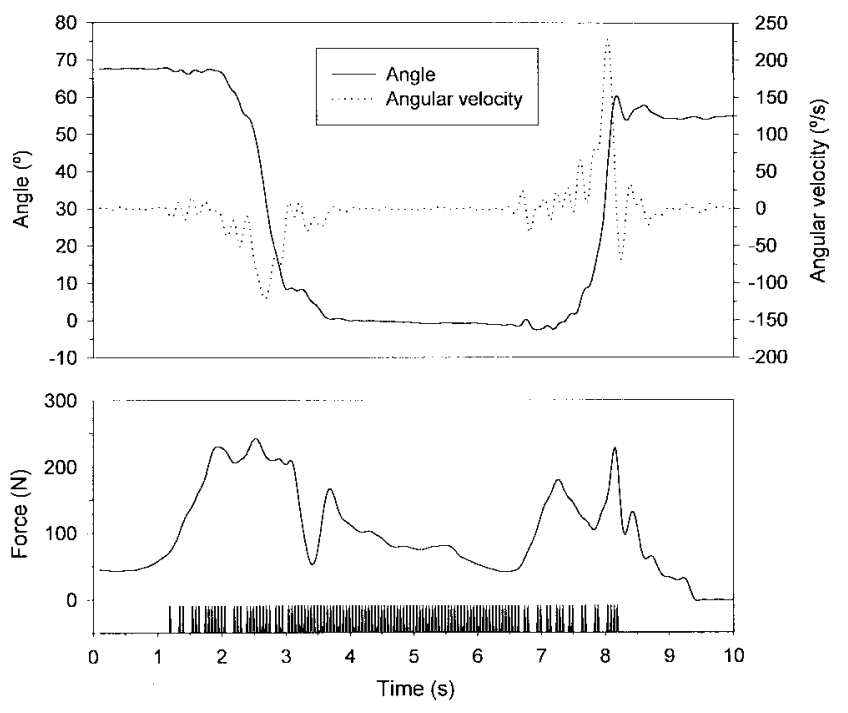

Fig. 4. Typical plots of the knee angle, knee angular velocity, hand support force and stimulation pulses against time for the SCC.

so that only small changes in the subject's position, as she attempted to initiate and terminate the maneuvers, caused the switching curve to be crossed repeatedly.

For standing up, the duration was significantly increased for the SCC (Table I), but examination of the knee angle trajectories suggest that this was due to the oscillations at the start and termination of the maneuver, rather than due to a more controlled ascent. The support force was significantly increased for the SCC, indicating that the subject needed to exert a greater voluntary effort than with the OLC. The duration of stimulation before standing up was negative, indicating that the subject must have anticipated the onset of stimulation. The comparisons of the termination velocities and the accelerations were inconclusive.

For sitting down, the stimulation duration demonstrated that with the OLC the subject started to descend only when the stimulation terminated (Table II). The subject was therefore effectively sitting down without the aid of ES and was relying on her upper body strength to control her descent. In contrast, with the SCC, the subject was assisted by ES during most of the period of actual descent (the knees were within $1^{\circ}$ of maximum flexion at $58.7 \pm 18.2 \%$ of the maneuver duration). The termination angular velocities were all significantly reduced for the SCC. The maximum angular velocity and the angular acceleration at maximum flexion were also generally reduced, particularly in the earlier trials before the onset of muscle fatigue, indicating that the SCC was better at controlling the rate of descent. Muscle fatigue was indicated by the deterioration in performance, most notable in standing up, between the first and last OLC sets. Nevertheless, this was not found to change the overall conclusions from the comparisons of the two controllers for either maneuver [2].

\section{Discussion ANd CONCLUding COMmEnTS}

The results obtained without stimulation demonstrate that independent standing, without either stimulation or a nonactive orthosis, was not a practical option for the subject. With stimulation the handsupport forces were greatly reduced, demonstrating the usefulness of FES.

For standing up, the tested version of the SCC did not offer any quantifiable advantages over the OLC, but was found to increase the average support force by $8.4 \%$ (Table I). In a previous study [14] using a similar closed-loop controller it was reported that a reduction in the knee-end velocity (KEV) to $40 \%$ of that using an open-loop controller was possible. However, the definition used for the KEV was such that it implicitly favored the closed-loop controller and the experiments were performed with the subject supine in an mechanical simulator using supramaximal quadriceps stimulation. The durations and maximum knee extension angular velocities are comparable to those reported elsewhere for able bodied individuals [2], [11]. The termination angular velocities are also comparable, however, the angular accelerations at full extension are 2-2.5 times those in able bodied individuals [2].

For sitting down, the SCC was measurably better at controlling the subject's descent than the OLC with the termination velocities being reduced by an average of $27 \%$ (Table II). The durations are comparable to those reported for able bodied individuals [2]. The maximum flexion angular velocities for the OLC were double those for able bodied individuals, whilst those obtained with the SCC were 1.7 times greater [2]. For both controllers, the termination angular velocities and the angular accelerations at full flexion were 2-3.5 times higher than those for able bodied individuals [2]. Thus the subject's descent was less controlled than would be expected for an able bodied individual. Importantly, the SCC was found to reduce the knee angular velocities and accelerations, but further refinements will be required to control fully the termination of the maneuver. It would also be of value to investigate ramping down the quadriceps stimulation over several seconds as this might improve the performance of the OLC.

The results of the pilot study are encouraging, though further subject tests are desirable, and suggest that there are a number of ways in which the SCC might be improved. The oscillations apparent at the start and termination of the maneuvers might be removed by introducing a deadband or proportional controller. During standing up the support forces might be reduced by more intense stimulation, i.e., supramaximal or frequency modulated stimulation, at the start of the maneuver and by stimulation of hip extensors, which can improve the coordination of the hip and knee joints [15]. The termination velocities during standing up might be reduced by stimulating the hamstrings. The termination of both maneuvers could 
be more controlled by increasing the slope of the switching curve in that region. Only one knee angle was used as a control input to the SCC which presupposes a symmetrical response to stimulation, however, it is questionable whether independent control of each leg is preferable when a symmetrical movement is desired.

The SCC has the advantage that it is generic, requiring no knowledge of the system plant, making it particularly easy to implement clinically. It is also computationally undemanding and could be implemented on a single chip microcontroller using miniature, robust and low-cost micromachined sensors, such as rate gyroscopes [16]. These advantages and its demonstrated ability to break the rate of descent during sitting down make it, the authors believe, worthy of further development and investigation.

\section{ACKNOWLEDGMENT}

The authors wish to thank the staff and patients of the Roessingh Rehabilitation Centre, Enschede, The Netherlands, and the staff and students of the Bioengineering Unit, University of Strathclyde, Glasgow, U.K., especially M. H. Granat, D. J. Maxwell, and J. P. Paul. At the time of the experimental work, M. J. Dolan was a postgraduate student in the Bioengineering Unit, University of Strathclyde.

\section{REFERENCES}

[1] M. W. Rodosky, T. P. Andriacchi, and G. B. J. Andersson, "The influence of chair height on lower limb mechanics during rising," $J$. Orthopaed. Res., vol. 7, pp. 266-271, 1989.

[2] M. J. Dolan, Restoration and Biomechanical Evaluation of Standing Up and Sitting Down in Paraplegics using Functional Electrical Stimulation, Ph.D. dissertation, Univ. Strathclyde, Glasgow, U.K., 1997, unpublished.

[3] T. Bajd, A. Kralj, and R. Turk, "Standing up a healthy subject and a paraplegic patient," J. Biomechan., vol. 15, pp. 1-10, 1982.
[4] A. Kralj, T. Bajd, R. Turk, J. Krajnik, and H. Benko, "Gait restoration in paraplegic patients: A feasibility demonstration using multichannel surface electrode FES," J. Rehab. Res. Develop., vol. 20, pp. 3-20, 1983.

[5] B. J. Andrews, R. H. Baxendale, R. Barnett, G. F. Phillips, T. Yamazaki, J. P. Paul, and P. A. Freeman, "A hybrid FES orthosis incorporating closed loop control and sensory feedback," J. Biomed. Eng., vol. 10, pp. 189-195, 1988

[6] R. J. Jaeger, G. M. Yarkony, and R. M. Smith, "Standing the spinal cord injured by electrical stimulation: Refinement of a protocol for clinical use," IEEE Trans. Biomed. Eng., vol. 36, pp. 720-728, 1989.

[7] B. J. Andrews, "Hybridorthese fur die Fortbevegung von Querschnittsgelahmten," Medizinisch-Orthopadische Technik, vol. 110, pp. 84-88, 1990 .

[8] D. J. Dandy, Essential Orthopaedics and Trauma, 2nd ed. London, U.K.: Churchill Livingstone, 1993.

[9] J. C. Bayley, T. P. Cochran, and C. B. Sledge, "The weight-bearing shoulder," J. Bone. Joint. Surg., vol. 69-A, pp. 676-678, 1987.

[10] S. Nuzik, R. Lamb, A. VanSant, and S. Hirt, "Sit-to-stand movement pattern-A kinematic study," Phys. Therapy, vol. 66, pp. 1708-1713, 1986.

[11] E. R. Ikeda, M. L. Schenkman, P. O. Riley, and W. A. Hodge, "Influence of age on dynamics of rising from a chair," Phys. Therapy, vol. 71, pp. 473-481, 1991.

[12] M. Schlecht, A. J. Mulder, H. J. Hermens, J. Vorsteveld, J. Cloostermans, and G. Zilvold, "Selection and training of patients for FES," in Proc. COMAC BME Conf. Restoration of Walking Aided by Functional Electrical Stimulation, 1987, pp. 75-78.

[13] W. S. Dorn and D. D. McCracken, Numerical Methods with Fortran IV: Case Studies. Chichester, U.K.: Wiley, 1972.

[14] A. J. Mulder, P. H. Veltink, and H. B. K. Boom, "On/off control in FES-induced standing up: A model study and experiments," Med. Biol. Eng. Comput., vol. 30, pp. 205-212, 1992.

[15] P. H. Veltink, B. M. Geerdink, B. F. J. M. Koopman, P. A. Huijing, and H. B. K. Boom, "Control of FES-assisted standing-up in paraplegics-The role of mono- and biarticular muscles," in Proc. IEEE Eng. Med. Biol. Soc. Annu. Int. Conf., 1994, pp. 444-445.

[16] B. J. Andrews and R. Williamson, "Joint motion sensors for FES: The GyroGoniometer,” in Proc. RESNA97, 1997, pp. 262-264. 